\title{
Passive antibody therapy in COVID-19
}

Jonathan Abraham (D) 1,2,3,4

For over a century, transfusion of convalescent plasma from recovered individuals has been tried as a therapeutic approach when a novel pathogen emerges. As the world awaits SARS-CoV-2 vaccines to be tested and safely deployed, the rapidity with which antiviral monoclonal antibodies can be isolated and engineered offers an attractive alternative option for passive immunization.

The spillover of emerging viruses from their animal reservoirs into human populations continuously threatens public health. Multiple coronaviruses have emerged to cause lethal viral pneumonias in humans: they include MERS-CoV, SARS-CoV and SARS-CoV-2. SARS-CoV-2, which causes COVID-19, emerged in late 2019 and has since caused a pandemic of unprecedented scale in recent history. Therapeutic and prophylactic interventions against COVID-19 are urgently needed.

The coronavirus spike (S) protein mediates cell surface receptor binding and fusion of the viral and host cell membranes. The $\mathrm{S}$ protein is a target for antiviral antibodies produced during natural infection and comprises two functional subunits, S1 and S2. The S1 subunits of SARS-CoV and SARS-CoV-2 contain a receptor-binding domain that binds to angiotensinconverting enzyme 2 (ACE2) on the surface of host cells. S2 contains a transmembrane anchor and mediates fusion of viral and host cell membranes after particles are internalized into acidified endosomes, although fusion at the cell surface can also occur in certain scenarios. Neutralizing antibodies could block viral entry by preventing the $S$ protein from binding to host cell receptors (for example, ACE2) or by preventing the conformational changes the $S$ protein undergoes to mediate membrane fusion (FIG. 1 a). Neutralizing antibodies could also mimic receptor binding and prematurely trigger fusogenic conformational changes in the $\mathrm{S}$ protein before it engages ACE2.

\section{Convalescent plasma therapy}

Passive immunization with convalescent plasma involves transfusing the acellular portion of blood from individuals who have recovered from an infection to persons who are infected or at risk of infection. Plasma donors are presumed to have developed an effective antibody response to the offending pathogen. The conferred immunity is short term.

Some of the most convincing data supporting the use of convalescent plasma in acute viral infection are from studies on Argentine haemorrhagic fever, an illness caused by Junin virus that carries a case fatality rate of $15-30 \%$. In a prospective study involving more than 80 cases of Argentine haemorrhagic fever, individuals received convalescent plasma pre-determined, in vitro, to have a range of neutralizing antibody titres. Transfusion of convalescent plasma with a high neutralizing antibody titre (dose adjusted per recipient body weight) was required for therapeutic effectiveness. No deaths were observed in the highest titre treatment group, which included 34 individuals ${ }^{1}$. A retrospective analysis defined the importance of providing the plasma within 8 days of the onset of illness. Convalescent plasma is now used routinely to treat Argentine haemorrhagic fever.

Transfusion of convalescent plasma did not show any benefit in Ebola virus disease during a recent outbreak ${ }^{2}$. However, the neutralizing titre of the infused convalescent plasma was later found to be low. A retrospective study of patients with SARS receiving therapy with steroids and the antiviral ribavirin showed that those also receiving convalescent plasma were discharged earlier from the hospital ${ }^{3}$. The neutralizing antibody titre of the infused plasma, however, was not standardized, and the comparator group remained on steroids, which could have confounded the outcome ${ }^{3}$.

In a recent prospective, non-controlled study involving individuals with severe COVID-19, Duan et al. ${ }^{4}$ transfused plasma with high-titre neutralizing activity from individuals who had recovered from COVID-19. Post-transfusion, recipients had a rapid increase in serum neutralizing antibody titres, had no detectable SARS-CoV-2 viral RNA in their blood at the time of sampling and improved clinically. Another study showed that convalescent plasma given with a median time of more than 20 days after viral shedding was first detected had an apparent effect on viral clearance but no effect on mortality ${ }^{5}$, suggesting that the timing of transfusion fell out of the therapeutic window.

The ongoing pandemic is an opportunity to perform randomized and controlled studies to support the use of convalescent plasma in the treatment of COVID-19. Ideally, such studies would include a group receiving convalescent plasma with pre-defined high-titre 


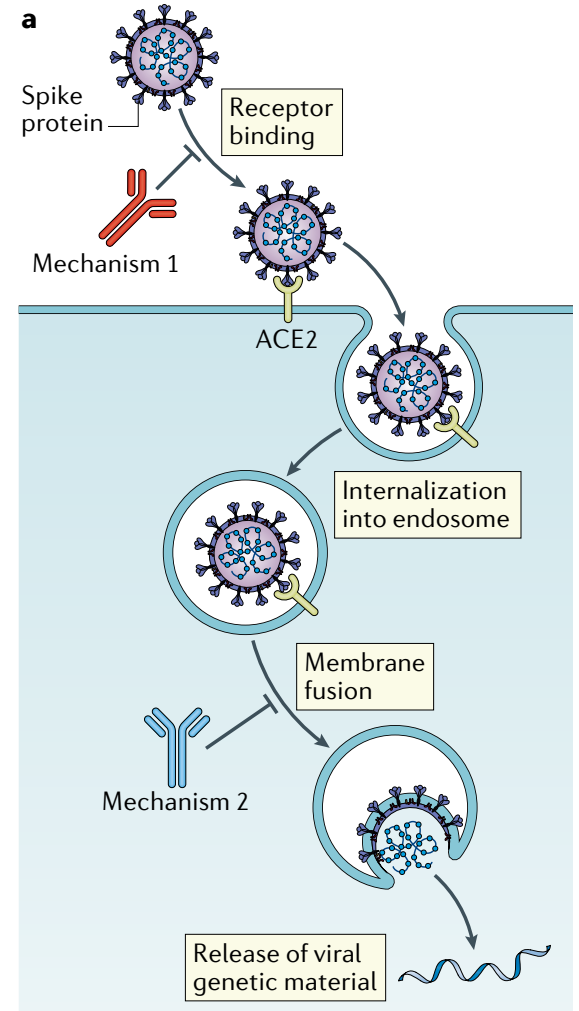

b

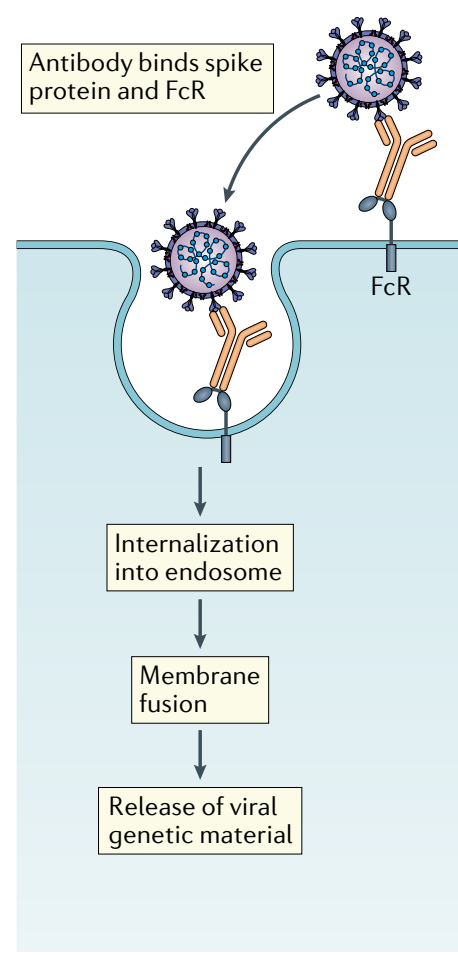

Fig. 1 | Potential mechanisms of coronavirus antibody neutralization and antibody enhancement of infection. a | Mechanism 1: neutralizing antibodies could block viral infection by binding to the viral spike protein and preventing it from interacting with the cellular receptor angiotensin-converting enzyme 2 (ACE2). Mechanism 2: neutralizing antibodies could bind to the viral spike protein and block the conformational changes that the spike protein must undergo to facilitate fusion of the viral and host cell membranes. $\mathbf{b} \mid$ Antibodies could enhance viral entry into immune cells by binding to the viral spike protein with their Fab portion and to Fc receptors (FcRs) with their Fc domain. virus B cell immortalization. The latter two approaches involve using blood samples obtained from recovered individuals. Monoclonal antibodies can also be rapidly scaled up for testing during outbreaks. Notable examples include $\mathrm{mAb} 114$, which comprises a single antibody, and REGN-EB3, a three-antibody cocktail. Both agents were shown to be effective against Ebola virus disease in a randomized clinical trial ${ }^{7}$.

Antibodies have two functional ends: their Fab arms interact with antigens, and their Fc domains interact with the adaptive and innate immune system, including natural killer cells, phagocytes and complement. Antibody Fc regions can be critical to the in vivo efficacy of passive immunization. Monoclonal antibodies, when developed for clinical applications, can be curated for several properties including the following: their neutralizing activity, the epitopes they target; and the antibody effector functions conferred by their $\mathrm{Fc}$ regions. To achieve the desired outcome and pharmacokinetics, there are antibody engineering opportunities for isotype or subclass switching, Fc glycan modification, or introduction of amino acid substitutions that modify Fc region affinity for Fc receptors (FcRs). Some antibodies might have undesirable effects, such as antibody-dependent enhancement (ADE) of infection of immune cells, including monocytes, macrophages and B cells (FIG. 1 b). ADE has been described for antibodies to the coronavirus $\mathrm{S}$ protein in vitro and in vivo for feline coronaviruses. ADE-promoting antibodies could be removed during therapeutic antibody candidate selection. Fc domains could also be modified to avoid ADE.

There are several reports of antibodies that potently neutralize SARS-CoV-2, including some that were isolated from COVID-19 convalescent donors that decrease viral RNA lung burden in animal models ${ }^{8,9}$. Such antibodies are poised for testing during this pandemic.

\section{Concluding remarks}

Critical questions include the following. What critical epitopes in the $\mathrm{S}$ protein are targeted by neutralizing antibodies found in convalescent plasma? How many neutralizing antibody epitopes can be targeted simultaneously on the SARS-CoV-2S protein? Which FcRs should be engaged by antibodies for optimal antiviral activity without exacerbating what might otherwise be an overexuberant immune response? Although coronaviruses have an exonuclease gene product that confers higher fidelity during genome replication, acquisition of antibody-escape mutations still remains a concern. Mutations that affect antibody neutralization, in principle, could occur and become fixed as the virus circulates during the pandemic. A cocktail of monoclonal antibodies, rather than a single agent, may decrease the likelihood of neutralization escape. Multiple vaccine platforms are now being tested with some non-human primate studies showing induction of SARS-CoV-2neutralizing antibodies ${ }^{10}$. Whether vaccine candidates will have varying efficacy in different subpopulations (such as older individuals) remains to be determined. Passive antibody administration may be a bridge to a vaccine in this vulnerable population, with the potential to be used for prophylaxis in particular settings 
(for instance, nursing homes). For all agents, appropriately controlled clinical trials, and the definition of an effective therapeutic window, are critical next steps.

1. Enria, D. A. et al. Importance of dose of neutralising antibodies in treatment of Argentine haemorrhagic fever with immune plasma. Lancet 2, 255-256 (1984).

2. van Griensven, J. et al. Evaluation of convalescent plasma for ebola virus disease in Guinea. N. Engl. J. Med. 374, 33-42 (2016).

3. Soo, Y. O. Y. et al. Retrospective comparison of convalescent plasma with continuing high-dose methylprednisolone treatment in SARS patients. Clin. Microbiol. Infect. 10, 676-678 (2004).

4. Duan, K. et al. Effectiveness of convalescent plasma therapy in severe COVID-19 patients. Proc. Natl Acad. Sci. USA 117 , 9490-9496 (2020).

5. Zeng, Q.-L. et al. Effect of convalescent plasma therapy on viral shedding and survival in patients with coronavirus disease 2019 J. Infect. Dis. https://doi.org/10.1093/infdis/jiaa228 (2020).
6. Lee, N. et al. Anti-SARS-CoV IgG response in relation to disease severity of severe acute respiratory syndrome. J. Clin. Virol. 35 , 179-184 (2006).

7. Levine, M. M. Monoclonal antibody therapy for ebola virus disease. N. Engl. J. Med. 381, 2365-2366 (2019).

8. Cao, Y. et al. Potent neutralizing antibodies against SARS-CoV-2 identified by high-throughput single-cell sequencing of convalescent patients' B cells. Cell https://doi.org/10.1016/j.cell.2020.05.025 (2020).

9. Rogers, T. F. et al. Rapid isolation of potent SARS-CoV-2 neutralizing antibodies and protection in a small animal model. Preprint at bioRxiv https://doi.org/10.1101/2020.05.11.088674 (2020).

10. Yu, J. et al. DNA vaccine protection against SARS-CoV-2 in rhesus macaques. Science https://doi.org/10.1126/science.abc6284 (2020).

\section{Competing interests}

The authors declare no competing interests. 